\title{
Stochastic Hopf-Hopf bifurcation of two-species discrete coupling logistic system with symbiotic interaction
}

\author{
Maosong Yang ${ }^{1,2}$ and Shaojuan $\mathrm{Ma}^{2 *}$
}

${ }^{*}$ Correspondence:
sjma.math@yahoo.com
${ }^{2}$ School of Mathematics and
Information Science, North Minzu
University, Yinchuan, China
Full list of author information is
available at the end of the article

available at the end of the article

\begin{abstract}
In this paper, stochastic Hopf-Hopf bifurcation of the discrete coupling logistic system with symbiotic interaction is investigated. Firstly, orthogonal polynomial approximation of discrete random function in the Hilbert spaces is applied to reduce the discrete coupling logistic system with random parameter to the deterministic equivalent system. Then, it is concluded that Hopf-Hopf bifurcation exists in the equivalent deterministic system according to the principle of algebraic criteria. Numerical simulations show that the bifurcation critical value varies with the intensity of random parameter, and Hopf-Hopf bifurcation and period-doubling bifurcation behavior exist. In particular, Hopf-Hopf bifurcation can be drift with the change of random intensity, and frequency locking phenomenon occurs in the stochastic system.
\end{abstract}

Keywords: Discrete coupling stochastic logistic system; Orthogonal polynomial approximation; Hopf-Hopf bifurcation; Random intensity

\section{Introduction}

In 1798, Malthus proposed the population growth model which stated that the world population grew geometrically as the food and space resources became more abundant. The mathematical ecologist May suggested in one of his influential articles published in 1976 by Nature [1] that the non-heterogamous insect population model in ecology can be explained in terms of the nonlinear difference equation

$$
x_{n+1}=\mu x_{n}\left(1-x_{n}\right) .
$$

We call Eq. (1) logistic map or logistic system. As we all know, Eq. (1) and the form of generalization were studied in detail in reference [2]. After that many researchers have studied the logistic system in mathematical ecology, social science, economic net, and so on [3-7]. At the same time people studied the logistic system further in more aspects. So, some references [8-10] have paid more attention to the dynamical behavior in the logistic system, such as bifurcation, chaos, and stability. Recently, the dynamical behavior in many complicated logistic systems, such as coupling logistic system, has been explored.

(c) The Author(s) 2020. This article is licensed under a Creative Commons Attribution 4.0 International License, which permits use, sharing, adaptation, distribution and reproduction in any medium or format, as long as you give appropriate credit to the original author(s) and the source, provide a link to the Creative Commons licence, and indicate if changes were made. The images or other third party material in this article are included in the article's Creative Commons licence, unless indicated otherwise in a credit line to the material. If material is not included in the article's Creative Commons licence and your intended use is not permitted by statutory regulation or exceeds the permitted use, you will need to obtain permission directly from the copyright holder. To view a copy of this licence, visit http://creativecommons.org/licenses/by/4.0/. 
Masoaller, Cavalcante, and Leite studied the delayed coupling of logistic maps [11]. The reference [12] analyzed synchronous chaos in the coupled system of two logistic maps. The symmetry breaking bifurcations in a circular chain of $N$ coupled logistic maps have been presented in reference [13]. Zhang and Wang studied the spatiotemporal chaos in mixed linear-nonlinear coupled logistic map [14]. Leonel Rocha and Abdel-Kaddous Taha studied the Allee effect on the dynamical behavior of a new class of generalized logistic maps [15]. Duygu Arugaslan and Leyla Guzel considered the logistic equation with piecewise constant argument of generalized type and analyzed the stability of the trivial fixed point and the positive fixed point after reducing the equation into a nonautonomous difference equation [16]. The dynamical properties of a stochastic susceptible-infected epidemic model with logistic growth were investigated in reference [17]. Qifa Lin performed stability analysis of a single species logistic model with Allee effect and feedback control [18]. On the other hand, these results are limited to a deterministic logistic system. As is known to all, in the real world, uncertainty of the system is inevitable, which can affect the dynamical behavior of the nonlinear system. Therefore the influence of random factors on the logistic system are worthy of exploring. The effects of randomness on chaos and order of coupled logistic maps have been stated by Marcelo [19]. The stochastic resonance problem for a class of time-delay logistics systems has been discussed [20]. Xing and Peng studied the boundedness, persistence, and extinction of a stochastic nonautonomous logistic system with time delays [21]. The reference [22] studied the dynamical behaviors of stochastic delay logistic system with impulsive toxicant in polluted environment. The stability of a stochastic logistic model with distributed delay has been explored in reference [23]. Xu Yong and Ma Shaojuan studied Hopf bifurcation control in a nonlinear stochastic dynamical system with nonlinear random feedback method [24]. Xu Yong et al. performed stability analysis in a stochastic Brusselator system with random parameter [25]. The reference [26] discussed a sliding mode control (SMC) for a wing model excited by harmonic forces and colored Gaussian noise together. JinZhong MA et al. introduced a periodically driven bistable eutrophication model with Gaussian white noise as a prototype class of real systems; they found that stochastic perturbations and periodic excitations were also able to slow down an imminent critical transition [27]. Qi Liu et al. analyzed the influences of random fluctuations on a two-degree-of-freedom (TDOF) airfoil model with viscoelastic terms [28]. Zhang Xiaoyu et al. considered the tipping behaviors of the thermoacoustic system with the time-varying parameters and the combined excitations of additive and multiplicative colored noises [29]. Although the research results on stochastic logistics system have been obtained, there are few studies on the influence of uncertainty on bifurcation. In addition, as far as we know, the influence of internal random parameters on the dynamic behavior of logistics system has not been found in the existing literature.

Motivated by the above discussions, this paper takes the coupled logistic system, which has a role of cohesion between one-dimensional and high-dimensional logistic model, as an example. By using the statistical characteristic of random variable, we build a stochastic coupled logistic system with random parameter. The influence of a random parameter in the two-species coupled logistic system with symbiotic interaction on the Hopf-Hopf bifurcation is studied by orthogonal polynomial approximation [30-36].

This paper is organized as follows. In Sect. 2, we transform the stochastic two-species coupling logistic system with symbiotic interaction with random parameter into its equivalent deterministic one by orthogonal polynomial approximation. We analyze the Hopf- 
Hopf bifurcation of the stochastic coupling logistic system in Sect. 3. The numerical simulations of Hopf-Hopf bifurcation about the two-species coupling logistic system with symbiotic interaction are shown in Sect. 4. Finally, conclusions are drawn in Sect. 5.

\section{Stochastic coupling logistic system with random parameter and its orthogonal polynomial approximation}

Consider a discrete coupling logistic model for the symbiotic interaction of the dynamic system [37]

$$
\left\{\begin{array}{l}
x_{n+1}=\mu\left(y_{n}\right) x_{n}\left(1-x_{n}\right), \\
y_{n+1}=\mu\left(x_{n}\right) y_{n}\left(1-y_{n}\right),
\end{array}\right.
$$

where $x_{n}, y_{n}$ are symbiotic species, the symbiotic interaction leads to both species that the growth rate $\mu(z)$ is varying with time. As it is seen in the equations, we call Eq. (2) symmetrical interaction. And it is shown that the particular dynamics of each species is a logistic map whose parameter $\mu_{n}$ is not fixed, $x_{n+1}=\mu x_{n}\left(1-x_{n}\right)$, but it is forced to remain in the interval [38-41]. The existence of a nontrivial fixed point at each step $n$ ensures the nontrivial evolution of the system [42]

$$
\mu(z)=\bar{u}(3 z+1), \quad \bar{u} \in(0,1.0843),
$$

where $u$ is a positive constant, and we call that the mutual benefit. At the same time, $\bar{u}$ is a random parameter which can be described as

$$
\bar{u}=u+\delta k,
$$

where $u$ is the deterministic system parameter of $\bar{u}, \delta$ is regarded as the intensity of random disturbance, $k$ is a random variable which obeys density function $p_{k}$ with standard deviation $\lambda$. By Eq. (2) and Eq. (3), we obtain

$$
\left\{\begin{array}{l}
x_{n+1}=\bar{u}\left(3 y_{n}+1\right) x_{n}\left(1-x_{n}\right) \\
y_{n+1}=\bar{u}\left(3 x_{n}+1\right) y_{n}\left(1-y_{n}\right) .
\end{array}\right.
$$

Obviously, there are many fixed points in this discrete coupling logistic system as we choose reasonable system variables. Origin $P_{0}(0,0)$ is a fixed point, other fixed points are: $P_{1}\left(\frac{\bar{u}-1}{\bar{u}}, 0\right), P_{2}\left(0, \frac{\bar{u}-1}{\bar{u}}\right), P_{3}\left(\frac{1}{3}\left(1+\sqrt{4-\frac{3}{\bar{u}}}\right), \frac{1}{3}\left(1+\sqrt{4-\frac{3}{\bar{u}}}\right)\right), P_{4}\left(\frac{1}{3}\left(1-\sqrt{4-\frac{3}{\bar{u}}}\right), \frac{1}{3}\left(1-\sqrt{4-\frac{3}{\bar{u}}}\right)\right)$. In order to compute simply, we select origin $P_{0}(0,0)$ to analyze the Hopf-Hopf bifurcation. So it follows from the orthogonal polynomial approximation that the response of Eq. (5) can be expressed by the following Fourier series under the condition of convergence in mean square:

$$
\left\{\begin{array}{l}
x(n, k)=\sum_{i=0}^{M} x_{i}(n) P_{i}^{\lambda}(k), \\
y(n, k)=\sum_{i=0}^{M} y_{i}(n) P_{i}^{\lambda}(k),
\end{array}\right.
$$

where $x_{i}(n)=\sum_{k=0}^{N} p_{k} x(n, k) P_{i}^{\lambda}(k), y_{i}(n)=\sum_{k=0}^{N} p_{k} y(n, k) P_{i}^{\lambda}(k), P_{i}^{\lambda}(k)$ is the $i$ th standard orthogonal polynomial, $M$ represents the largest order of the polynomial we have taken. 
Substituting Eq. (6) and Eq. (4) into Eq. (5), we obtain

$$
\left\{\begin{aligned}
\sum_{i=0}^{M} x_{i}(n+1) P_{i}^{\lambda}(k) \\
=3 u\left[\sum_{i=0}^{M} x_{i}(n) P_{i}^{\lambda}(k)\right]\left[\sum_{i=0}^{M} y_{i}(n) P_{i}^{\lambda}(k)\right]+u \sum_{i=0}^{M} x_{i}(n) P_{i}^{\lambda}(k) \\
+3 \delta k\left[\sum_{i=0}^{M} x_{i}(n) P_{i}^{\lambda}(k)\right]\left[\sum_{i=0}^{M} y_{i}(n) P_{i}^{\lambda}(k)\right]+\delta k \sum_{i=0}^{M} x_{i}(n) P_{i}^{\lambda}(k) \\
-3 u\left[\sum_{i=0}^{M} x_{i}(n) P_{i}^{\lambda}(k)\right]^{2}\left[\sum_{i=0}^{M} y_{i}(n) P_{i}^{\lambda}(k)\right]-u\left[\sum_{i=0}^{M} x_{i}(n) P_{i}^{\lambda}(k)\right]^{2} \\
-3 \delta k\left[\sum_{i=0}^{M} x_{i}(n) P_{i}^{\lambda}(k)\right]^{2}\left[\sum_{i=0}^{M} y_{i}(n) P_{i}^{\lambda}(k)\right]-\delta k\left[\sum_{i=0}^{M} x_{i}(n) P_{i}^{\lambda}(k)\right]^{2}, \\
\sum_{i=0}^{M} y_{i}(n+1) P_{i}^{\lambda}(k) \\
=3 u\left[\sum_{i=0}^{M} x_{i}(n) P_{i}^{\lambda}(k)\right]\left[\sum_{i=0}^{M} y_{i}(n) P_{i}^{\lambda}(k)\right]+u \sum_{i=0}^{M} y_{i}(n) P_{i}^{\lambda}(k) \\
+3 \delta k\left[\sum_{i=0}^{M} x_{i}(n) P_{i}^{\lambda}(k)\right]\left[\sum_{i=0}^{M} y_{i}(n) P_{i}^{\lambda}(k)\right]+\delta k \sum_{i=0}^{M} y_{i}(n) P_{i}^{\lambda}(k) \\
-3 u\left[\sum_{i=0}^{M} y_{i}(n) P_{i}^{\lambda}(k)\right]^{2}\left[\sum_{i=0}^{M} x_{i}(n) P_{i}^{\lambda}(k)\right]-u\left[\sum_{i=0}^{M} y_{i}(n) P_{i}^{\lambda}(k)\right]^{2} \\
-3 \delta k\left[\sum_{i=0}^{M} y_{i}(n) P_{i}^{\lambda}(k)\right]^{2}\left[\sum_{i=0}^{M} x_{i}(n) P_{i}^{\lambda}(k)\right]-\delta k\left[\sum_{i=0}^{M} y_{i}(n) P_{i}^{\lambda}(k)\right]^{2} .
\end{aligned}\right.
$$

With the aid of a cycle recurrence formula of orthogonal polynomial [43]

$$
k P_{i}^{\lambda}(k)=\alpha_{i} P_{i+1}^{\lambda}(k)+\beta_{i} P_{i}^{\lambda}(k)+\gamma_{i} P_{i-1}^{\lambda}(k),
$$

the nonlinearity term in the right equation of Eq. (7) can be respectively written as follows:

$$
\left[\sum_{i=0}^{M} x_{i}(n) P_{i}^{\lambda}(k)\right]\left[\sum_{i=0}^{M} y_{i}(n) P_{i}^{\lambda}(k)\right]=\sum_{i=0}^{2 M} S_{i}(n) P_{i}^{\lambda}(k),
$$

and

$$
\left[\sum_{i=0}^{M} x_{i}(n) P_{i}^{\lambda}(k)\right]^{2}\left[\sum_{i=0}^{M} y_{i}(n) P_{i}^{\lambda}(k)\right]=\sum_{i=0}^{3 M} W_{i}(n) P_{i}^{\lambda}(k),
$$

and

$$
\left[\sum_{i=0}^{M} x_{i}(n) P_{i}^{\lambda}(k)\right]^{2}=\sum_{i=0}^{2 M} X_{i}(n) P_{i}^{\lambda}(k)
$$

and

$$
\left[\sum_{i=0}^{M} y_{i}(n) P_{i}^{\lambda}(k)\right]^{2}\left[\sum_{i=0}^{M} x_{i}(n) P_{i}^{\lambda}(k)\right]=\sum_{i=0}^{3 M} Z_{i}(n) P_{i}^{\lambda}(k),
$$

and

$$
\left[\sum_{i=0}^{M} y_{i}(n) P_{i}^{\lambda}(k)\right]^{2}=\sum_{i=0}^{2 M} Y_{i}(n) P_{i}^{\lambda}(k)
$$


The stochastic term in the right equation of Eq. (7) can be respectively written as follows:

$$
\begin{aligned}
\delta k & {\left[\sum_{i=0}^{M} x_{i}(n) P_{i}^{\lambda}(k)\right]\left[\sum_{i=0}^{M} y_{i}(n) P_{i}^{\lambda}(k)\right] } \\
& =\delta \sum_{i=0}^{2 M}\left[P_{i}^{\lambda}(k)\left(\gamma_{i} S_{i+1}(n)+\beta_{i} S_{i}(n)+\alpha_{i} S_{i-1}(n)\right)-\alpha_{i} S_{i}(n) P_{i+1}^{\lambda}(k)\right],
\end{aligned}
$$

and

$$
\begin{aligned}
\delta k & \sum_{i=0}^{M} x_{i}(n) P_{i}^{\lambda}(k) \\
\quad & \delta \sum_{i=0}^{M}\left[P_{i}^{\lambda}(k)\left(\gamma_{i} x_{i+1}(n)+\beta_{i} x_{i}(n)+\alpha_{i} x_{i-1}(n)\right)-\alpha_{i} x_{i}(n) P_{i+1}^{\lambda}(k)\right],
\end{aligned}
$$

and

$$
\begin{aligned}
\delta k & {\left[\sum_{i=0}^{M} x_{i}(n) P_{i}^{\lambda}(k)\right]^{2} } \\
& =\delta \sum_{i=0}^{2 M}\left[P_{i}^{\lambda}(k)\left(\gamma_{i} X_{i+1}(n)+\beta_{i} X_{i}(n)+\alpha_{i} X_{i-1}(n)\right)-\alpha_{i} X_{i}(n) P_{i+1}^{\lambda}(k)\right],
\end{aligned}
$$

and

$$
\begin{aligned}
\delta k & {\left[\sum_{i=0}^{M} x_{i}(n) P_{i}^{\lambda}(k)\right]^{2}\left[\sum_{i=0}^{M} y_{i}(n) P_{i}^{\lambda}(k)\right] } \\
& =\delta \sum_{i=0}^{3 M}\left[P_{i}^{\lambda}(k)\left(\gamma_{i} W_{i+1}(n)+\beta_{i} W_{i}(n)+\alpha_{i} W_{i-1}(n)\right)-\alpha_{i} W_{i}(n) P_{i+1}^{\lambda}(k)\right],
\end{aligned}
$$

and

$$
\begin{aligned}
\delta k & \sum_{i=0}^{M} y_{i}(n) P_{i}^{\lambda}(k) \\
& =\delta \sum_{i=0}^{M}\left[P_{i}^{\lambda}(k)\left(\gamma_{i} y_{i+1}(n)+\beta_{i} y_{i}(n)+\alpha_{i} y_{i-1}(n)\right)-\alpha_{i} y_{i}(n) P_{i+1}^{\lambda}(k)\right],
\end{aligned}
$$

and

$$
\begin{aligned}
& \delta k\left[\sum_{i=0}^{M} y_{i}(n) P_{i}^{\lambda}(k)\right]^{2} \\
& =\delta \sum_{i=0}^{2 M}\left[P_{i}^{\lambda}(k)\left(\gamma_{i} Y_{i+1}(n)+\beta_{i} Y_{i}(n)+\alpha_{i} Y_{i-1}(n)\right)-\alpha_{i} Y_{i}(n) P_{i+1}^{\lambda}(k)\right],
\end{aligned}
$$


and

$$
\begin{aligned}
& \delta k\left[\sum_{i=0}^{M} y_{i}(n) P_{i}^{\lambda}(k)\right]^{2}\left[\sum_{i=0}^{M} x_{i}(n) P_{i}^{\lambda}(k)\right] \\
& =\delta \sum_{i=0}^{3 M}\left[P_{i}^{\lambda}(k)\left(\gamma_{i} Z_{i+1}(n)+\beta_{i} Z_{i}(n)+\alpha_{i} Z_{i-1}(n)\right)-\alpha_{i} Z_{i}(n) P_{i+1}^{\lambda}(k)\right],
\end{aligned}
$$

where $S_{i}(n), W_{i}(n), X_{i}(n), Z_{i}(n)$, and $Y_{i}(n)$ stand for the linear combination of nonlinearity terms calculated by computer algebraic, such as Maple. According to Eqs. (9)-(20), we obtain that Eq. (7) can be further reduced to

$$
\left\{\begin{aligned}
\sum_{i=0}^{M} & x_{i}(n+1) P_{i}^{\lambda}(k) \\
= & u \sum_{i=0}^{2 M} S_{i}(n) P_{i}^{\lambda}(k)+u \sum_{i=0}^{M} x_{i}(n) P_{i}^{\lambda}(k)+3 \delta \sum_{i=0}^{2 M}\left[P _ { i } ^ { \lambda } ( k ) \left(\lambda(i+1) S_{i+1}(n)\right.\right. \\
& \left.\left.+(i+\lambda) S_{i}(n)+S_{i-1}(n)\right)-S_{i}(n) P_{i+1}^{\lambda}(k)\right] \\
& +\delta \sum_{i=0}^{M}\left[P _ { i } ^ { \lambda } ( k ) \left(\lambda(i+1) x_{i+1}(n)+(i+\lambda) x_{i}(n)\right.\right. \\
& \left.\left.\left.+x_{i-1}(n)\right)-x_{i}(n) P_{i+1}^{\lambda}(k)\right]-3 u \sum_{i=0}^{3 M} W_{i}(n) P_{i}^{\lambda}(k)\right] \\
& -u \sum_{i=0}^{2 M} X_{i}(n) P_{i}^{\lambda}(k)-3 \delta \sum_{i=0}^{3 M}\left[P_{i}^{\lambda}(k)(\lambda(i+1)\right. \\
& \left.\left.\times W_{i+1}(n)+(i+\lambda) W_{i}(n)+W_{i-1}(n)\right)-W_{i}(n) P_{i+1}^{\lambda}(k)\right] \\
& -\delta \sum_{i=0}^{2 M}\left[P _ { i } ^ { \lambda } ( k ) \left(\lambda(i+1) X_{i+1}(n)+(i+\lambda)\right.\right. \\
& \left.\left.\times X_{i}(n)+X_{i-1}(n)\right)-X_{i}(n) P_{i+1}^{\lambda}(k)\right], \\
\sum_{i=0}^{M} & y_{i}(n+1) P_{i}^{\lambda}(k) \\
= & 3 u \sum_{i=0}^{2 M} S_{i}(n) P_{i}^{\lambda}(k)+u \sum_{i=0}^{M} y_{i}(n) P_{i}^{\lambda}(k)+3 \delta \sum_{i=0}^{2 M}\left[P _ { i } ^ { \lambda } ( k ) \left(\lambda(i+1) S_{i+1}(n)\right.\right. \\
& \left.\left.+(i+\lambda) S_{i}(n)+S_{i-1}(n)\right)-S_{i}(n) P_{i+1}^{\lambda}(k)\right] \\
& +\delta \sum_{i=0}^{M}\left[P _ { i } ^ { \lambda } ( k ) \left(\lambda(i+1) y_{i+1}(n)+(i+\lambda) y_{i}(n)\right.\right. \\
& \left.\left.\left.+y_{i-1}(n)\right)-y_{i}(n) P_{i+1}^{\lambda}(k)\right]-3 u \sum_{i=0}^{3 M} Z_{i}(n) P_{i}^{\lambda}(k)\right] \\
& -u \sum_{i=0}^{2 M} Y_{i}(n) P_{i}(k)-3 \delta \sum_{i=0}^{3 M}\left[P_{i}^{\lambda}(k)(\lambda(i+1)\right. \\
& \left.\left.\times Z_{i+1}(n)+(i+\lambda) Z_{i}(n)+Z_{i-1}(n)\right)-Z_{i}(n) P_{i+1}^{\lambda}(k)\right] \\
& -\delta \sum_{i=0}^{2 M}\left[P _ { i } ^ { \lambda } ( k ) \left(\lambda(i+1) Y_{i+1}(n)+(i+\lambda)\right.\right. \\
& \left.\left.\times Y_{i}(n)+Y_{i-1}(n)\right)-Y_{i}(n) P_{i+1}^{\lambda}(k)\right],
\end{aligned}\right.
$$

where $x_{-1}(n), x_{M+1}, y_{-1}(n), y_{M+1}, S_{-1}(n), S_{M+1}(n), X_{-1}(n), X_{M+1}(n), Y_{-1}(n), Y_{M+1}(n), W_{-1}(n)$, $W_{M+1}(n), Z_{-1}(n)$, and $Z_{M+1}(n)$ are zero by the principle of approximation. We choose the random variable in compliance with Poisson distribution with standard deviation $\lambda$. According to the weight function, the weight orthogonal polynomial in Eq. (8) is chosen as Charlier polynomial. The coefficients $\alpha_{i}, \beta_{i}, \gamma_{i}$ are $1, i+\lambda, \lambda i$, respectively. Multiply both sides of Eq. (21) by $P_{i}^{\lambda}(k), i=0,1, \ldots, M$, in sequence and take expectation with respect to $k$, where $P_{i}^{\lambda}(k)=\frac{Q_{i}(k)}{\sqrt{\delta_{i}}}$ after standardization of polynomial. According to the statistical characteristics and orthogonal polynomial approximation of discrete random function in the Hilbert spaces and the orthogonality of Charlier orthogonal polynomials, we can finally get the equivalent deterministic logistic equation. As $M \rightarrow \infty$, the discrete coupling stochastic logistic system is strictly equivalent to system (21) in the sense of mean square convergence. In order to facilitate the numerical analysis of this paper, we select $M=1$, 
$\lambda=0.5$ and approximately obtain the equivalent deterministic system

$$
\left\{\begin{aligned}
x_{0}(n+1) & =3 u S_{0}(n)+u x_{0}(n)+\frac{3}{2} \delta\left[S_{1}(n)+S_{0}(n)\right]+\frac{1}{2} \delta\left[x_{1}(n)+x_{0}(n)\right] \\
& -3 u W_{0}(n)-u X_{0}(n)-\frac{3}{2} \delta\left[W_{1}(n)+W_{0}(n)\right]-\frac{1}{2} \delta\left[X_{1}(n)+X_{0}(n)\right], \\
y_{0}(n+1) & =3 u S_{0}(n)+u y_{0}(n)+\frac{3}{2} \delta\left[S_{1}(n)+S_{0}(n)\right]+\frac{1}{2} \delta\left[y_{1}(n)+y_{0}(n)\right] \\
& -3 u Z_{0}(n)-u Y_{0}(n)-\frac{3}{2} \delta\left[Z_{1}(n)+Z_{0}(n)\right]-\frac{1}{2} \delta\left[Y_{1}(n)+Y_{0}(n)\right], \\
x_{1}(n+1) & \\
= & 3 u S_{1}(n)+u x_{1}(n)+3 \delta\left[\frac{3}{2} S_{1}(n)-S_{0}(n)\right]+\delta\left[\frac{3}{2} x_{1}(n)-x_{0}(n)\right] \\
& -3 u W_{1}(n)-u X_{1}(n)-3 \delta\left[\frac{3}{2} W_{1}(n)-W_{0}(n)\right]-\delta\left[\frac{3}{2} X_{1}(n)-X_{0}(n)\right], \\
y_{1}(n+1) & =3 u S_{1}(n)+u y_{1}(n)+3 \delta\left[\frac{3}{2} S_{1}(n)-S_{0}(n)\right]+\delta\left[\frac{3}{2} y_{1}(n)-y_{0}(n)\right] \\
& -3 u Z_{1}(n)-u Y_{1}(n)-3 \delta\left[\frac{3}{2} Z_{1}(n)-Z_{0}(n)\right]-\delta\left[\frac{3}{2} Y_{1}(n)-Y_{0}(n)\right] .
\end{aligned}\right.
$$

Then the approximate random response of the original stochastic logistic system can be expressed as

$$
\left\{\begin{array}{l}
x(n, k)=\sum_{i=0}^{1} x_{i}(n) P_{i}^{\lambda}(k)=\sum_{i=0}^{1} x_{i}(n) \frac{Q_{i}(k)}{\sqrt{\delta_{i}}} \\
y(n, k)=\sum_{i=0}^{1} y_{i}(n) P_{i}^{\lambda}(k)=\sum_{i=0}^{1} y_{i}(n) \frac{Q_{i}(k)}{\sqrt{\delta_{i}}}
\end{array}\right.
$$

and as $k=0$, the sample response of mean parameter system (SMR) and the ensemble mean response of it (EMR) are calculated as follows:

$$
\left\{\begin{array}{l}
E[x(n, k)]=\sum_{i=0}^{1} x_{i}(n) E\left[P_{i}^{\lambda}(k)\right]=x_{0}(n) E\left[P_{0}^{\lambda}(k)\right]+x_{1}(n) E\left[P_{1}^{\lambda}(k)\right] \\
E[y(n, k)]=\sum_{i=0}^{1} y_{i}(n) E\left[P_{i}^{\lambda}(k)\right]=y_{0}(n) E\left[P_{0}^{\lambda}(k)\right]+y_{1}(n) E\left[P_{1}^{\lambda}(k)\right] .
\end{array}\right.
$$

In this paper, we take the initial conditions of deterministic equivalent system (22) and the same initial conditions of the deterministic system

$$
x_{0}=x_{0}(0)=0.1, \quad y_{0}=y_{0}(0)=0.2, \quad x_{1}=x_{1}(0)=0.1, \quad y_{1}=y_{1}(0)=0 \text {. }
$$

So we take

$$
x(0)=(0.1,0.1)^{T}, \quad y(0)=(0.2,0)^{T} .
$$

\section{Hopf bifurcation analysis}

We firstly introduce the Hopf-Hopf bifurcation conditions [44] about the deterministic discrete system as follows.

Lemma 1 For the map $x \rightarrow F(x, \mu), x \in R^{n}, \mu \in R^{m}$, if the eigenvalues of a Jacobian matrix at the bifurcation parameter point $\mu=\mu_{c}$, we have the following properties: 
(1) There are two pairs of complex conjugate eigenvalues on the unit circle, which is marked by $\left|\lambda_{1,2}\left(\mu_{c}\right)\right|=1$ with $\lambda_{1}=\overline{\lambda_{2}}$ and $\left|\lambda_{3,4}\left(\mu_{c}\right)\right|=1$ with $\lambda_{3}=\overline{\lambda_{4}}$, and others are inside the unit circle (i.e., $\left.\left|\lambda_{j}\left(\mu_{0}\right)\right|<1, j=5,6, \ldots, n\right)$;

(2) Transversality condition $\frac{d\left|\lambda_{j}\left(\mu_{c}\right)\right|}{d \mu} \neq 0(j=1,3, k=1,2, \ldots, m)$,

(3) Nonresonance condition $\lambda_{1}^{m}\left(\mu_{c}\right) \neq 1$ and $\lambda_{3}^{m}\left(\mu_{c}\right) \neq 1, m=3,4, \ldots$

Then a nonresonant Hopf-Hopf bifurcation of the map $x \rightarrow F(x, \mu), x \in R^{n}, \mu \in R^{m}$, occurs at $\mu=\mu_{c}$.

Let us assume that the solutions to the deterministic equivalent discrete coupling logistic system undergo a Hopf-Hopf bifurcation on some submanifold in parameter space corresponding to a critical value $\bar{\mu}=\bar{\mu}_{c}$.

The Jacobian matrix of system $(22)$ at fixed point $P_{0}(0,0)$ is

$$
A=\left(\begin{array}{cccc}
u+\frac{1}{2} \delta & 0 & \frac{1}{2} \delta & 0 \\
0 & u+\frac{1}{2} \delta & 0 & \frac{1}{2} \delta \\
-\delta & 0 & u+\frac{3}{2} \delta & 0 \\
0 & -\delta & 0 & u+\frac{3}{2} \delta
\end{array}\right)
$$

Then the characteristic polynomial of Jacobian matrix $A$ is

$$
f(z)=z^{4}+a_{1} z^{3}+a_{2} z^{2}+a_{3} z+a_{4}
$$

where $a_{i}(i=1,2,3,4)$ are coefficients of the characteristic equation, which are shown as follows:

$$
\begin{aligned}
& a_{1}=4 u-4 \delta \\
& a_{2}=\frac{13}{2} \delta^{2}+6 u^{2}+12 u \delta \\
& a_{3}=-13 u \delta^{2}-5 \delta^{3}-4 u^{3}-12 u^{2} \delta \\
& a_{4}=\frac{13}{2} u^{2} \delta^{2}+5 u \delta^{3}+\frac{25}{16} \delta^{4}+u^{4}+4 u^{3} \delta
\end{aligned}
$$

By the mathematical software, all eigenvalues of Eq. (25) are $z_{1,2}=(u+\delta) \pm \frac{1}{2} \delta i, z_{3,4}=$ $(u+\delta) \pm \frac{1}{2} \delta i\left(i^{2}=-1\right)$.

Obviously Eq. (25) has a pair of conjugate complex roots, Hopf bifurcation will occur in system (22).

Now, discussing when the eigenvalues module $\left|z_{1}\right|=\left|z_{2}\right|=\left|z_{3}\right|=\left|z_{4}\right|=1$, we can get the relations between the bifurcation parameter and the random strength:

$$
u_{1}=-\delta+\sqrt{1-\frac{1}{4} \delta^{2}}, \quad u_{2}=-\delta-\sqrt{1-\frac{1}{4} \delta^{2}}
$$

Due to the random strength $\delta>0$ and $u>0$ when $\delta=0$, there is only one equation $u_{c}=$ $u_{1}=-\delta+\sqrt{1-\frac{1}{4} \delta^{2}}$ satisfying $-2<\delta<2$, eigenvalues of Eq. (25) can satisfy eigenvalues module is equal to 1 . Then the expression $u=u_{c}$ is substituted into characteristic Eq. (25), 
and we obtain all eigenvalues as follows:

$$
z_{1,2}=\sqrt{1-\frac{1}{4} \delta^{2}} \pm \frac{1}{2} \delta i, \quad z_{3,4}=\sqrt{1-\frac{1}{4} \delta^{2}} \pm \frac{1}{2} \delta i .
$$

Obviously, the Hopf-Hopf bifurcation condition (1) about system (22) is satisfied. We have

$$
\frac{d\left|z_{1}\left(u_{c}\right)\right|}{d u}=\left.\frac{(\delta+u)}{\sqrt{\frac{5}{4} \delta^{2}+2 \delta u+u^{2}}}\right|_{u=u_{c}}=\sqrt{1-\frac{1}{4} \delta^{2}}
$$

because $\delta>0$, when only $\delta \neq 2$, we have $\frac{d\left|z_{1}\left(u_{c}\right)\right|}{d u} \neq 0$, meanwhile $z_{1}^{m}\left(\mu_{c}\right) \neq 1$. The Hopf-Hopf bifurcation conditions (2) and (3) about system (22) are satisfied. The following theorem gives criterion that guarantees that the conditions for Hopf-Hopf bifurcation are satisfied.

Theorem 1 If $u_{c}$ is a bifurcation parameter of two-species discrete coupling stochastic logistic system with symbiotic interaction, $z_{1,2}=\sqrt{1-\frac{1}{4} \delta^{2}} \pm \frac{1}{2} \delta i, \frac{d\left|z_{1}\left(u_{c}\right)\right|}{d u}=\sqrt{1-\frac{1}{4} \delta^{2}}$, and $z_{1}^{m}\left(\mu_{c}\right) \neq 1$, then the conditions for the existence of a Hopf-Hopf bifurcation are satisfied.

It is to say that equivalent deterministic system (22) undergoes a Hopf-Hopf bifurcation at the equilibrium $(0,0)$. The above analysis is summarized as follows.

Theorem 2 (Existence of Hopf bifurcation) The two-species discrete coupling stochastic logistic system with symbiotic interaction undergoes the Hopf-Hopf bifurcation at the fixed point $(0,0)$ when the system parameter $u$ goes by the critical value $u_{c}=-\delta+\sqrt{1-\frac{1}{4} \delta^{2}}$. Specially, when the strength of random disturbance $\delta=0$, we have $u_{c}=1$, eigenvalues of Eq. (25) are $z_{1,2}=1$ and Hopf-Hopf bifurcation is $u=1$.

\section{The numerical simulation and numerical analysis}

In this section, when the strength of random intensity $\delta=0.000$, the discrete coupling stochastic logistic system with symbiotic interaction is investigated. System (5) can be turned into a deterministic original coupling logistic system. We can know this bifurcation parameter $u_{c}=1$, system (5) undergoes a Hopf-Hopf bifurcation at the fixed point $(0,0)$. Figure 1 shows each of the bifurcation phenomena about the coupling stochastic logistic system with symbiotic interaction, when the random intensity $\delta=0.000,0.005,0.020,0.240$. By the numerical analysis, the critical value for Hopf-Hopf bifurcation of the coupling stochastic logistic system with symbiotic interaction is $u_{c}=$ $-\delta+\sqrt{1-\frac{1}{4} \delta^{2}}$. At the same time, the Hopf-Hopf bifurcation under the influence of different random strength is in advance.

When $u=0.965$ and random intensity $\delta$ is equal to $0.000,0.002,0.006,0.010,0.050$, $0.090,0.150,0.200,0.240$, respectively, the phase trajectories of DR and EMR all converge to zero. It is shown in Fig. 2(a) and Fig. 2(b) that fixed points are stable with discrete two-species coupled logistic system with symbiotic interaction, mean as $\delta=0.000$ with two-species discrete coupling stochastic logistic system with symbiotic interaction, and Fig. 2(b) is a partial enlarged effect of Fig. 2(a). We know that the two-species discrete coupling stochastic logistic system with symbiotic interaction does not undergo Hopf-Hopf bifurcation, which is illustrated by Fig. 2(a). Increasing the random intensity to $\delta=0.002$, 


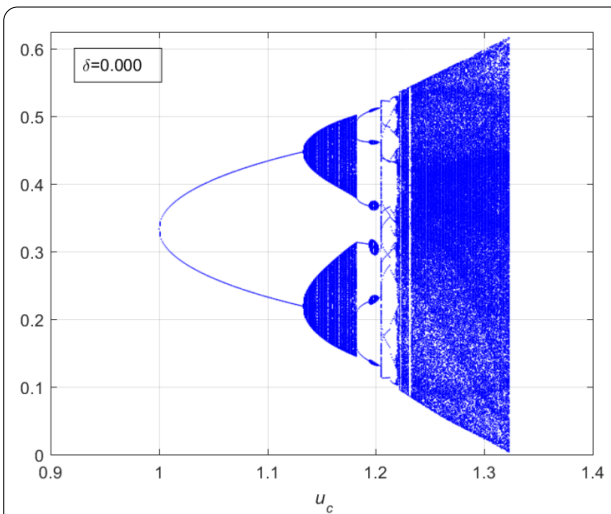

(a)

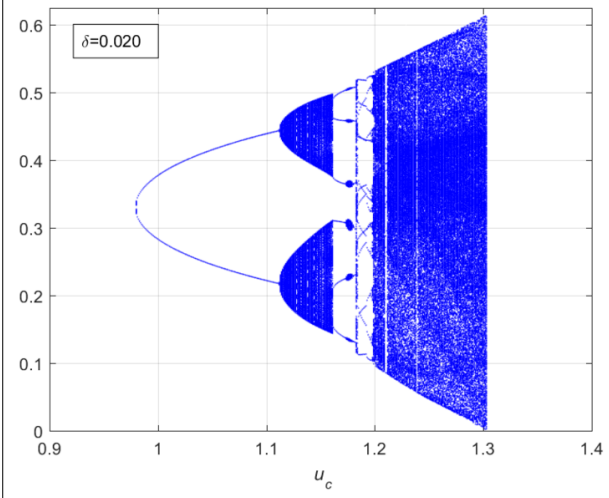

(c)

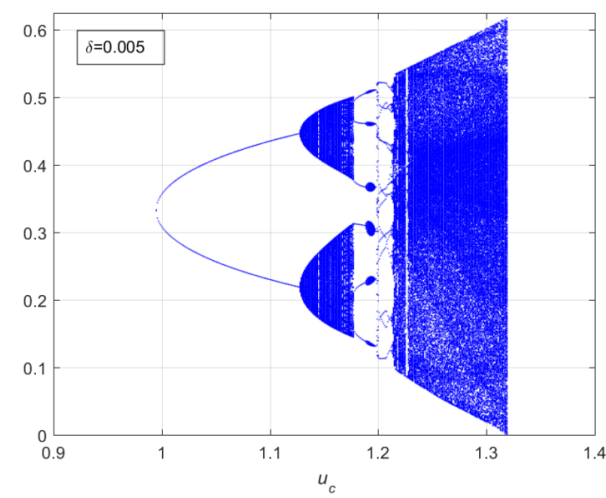

(b)

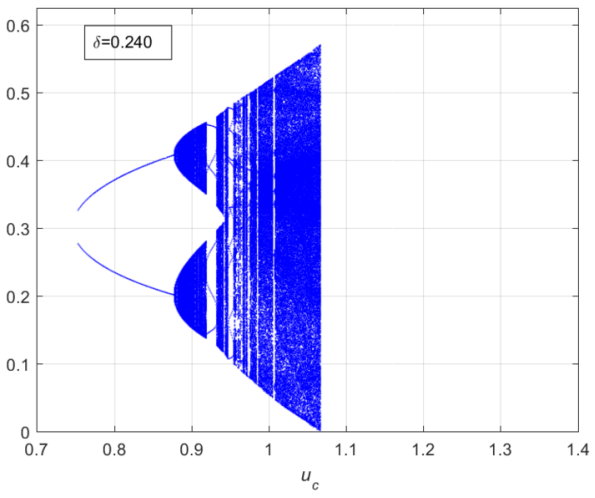

(d)

Figure 1 The bifurcation diagrams of deterministic and coupling stochastic logistic system

the phase trajectories of system (22) that converge to limit circle are shown in Fig. 2(c), (d), and Fig. 2(d) is a partial enlarged effect of Fig. 2(c). From Fig. 2(c), (e), (g), (i), (j), we find that with the change of random intensity, the amplitude of limit circle is increased. From Fig. $2(\mathrm{k})$ we can see that the limit circle happened to intersect when random intensity increased to $\delta=0.150$. At the same time, from Fig. 2(l), (m), (n), Fig. 2(f) is a partial enlarged effect of Fig. 2(e), and Fig. 2(h) is a partial enlarged effect of Fig. 2(g), Fig. 2(o) is a partial enlarged effect of Fig. 2(n). We can know that with the increase of random intensity the phase trajectories show random motion, and frequency lock phenomena appear at last. We find that with the change of bifurcation parameter the phase trajectories of the deterministic system accord with the phase trajectories of two-species discrete coupling stochastic logistic system with symbiotic interaction, the bifurcation happens in both systems. According to the above numerical analysis, we discover that the critical value for Hopf-Hopf bifurcation in the two-species discrete coupling stochastic logistic system with symbiotic interaction is varying from random intensity.

Based on the theoretical analysis and numerical simulations, we find that the HopfHopf bifurcation occurs in two-species discrete coupling stochastic logistic system with symbiotic interaction with the variation of bifurcation parameter. Compared with the deterministic system, the random intensity has an obvious influence on the bifurcation critical value of its coupled random logic system, and the bifurcation critical value decreases with the increase of random intensity, indicating that the Hopf-Hopf bifurcation point 


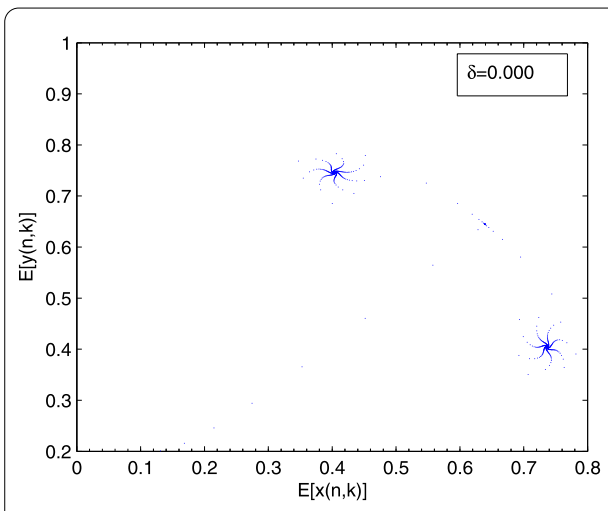

(a)

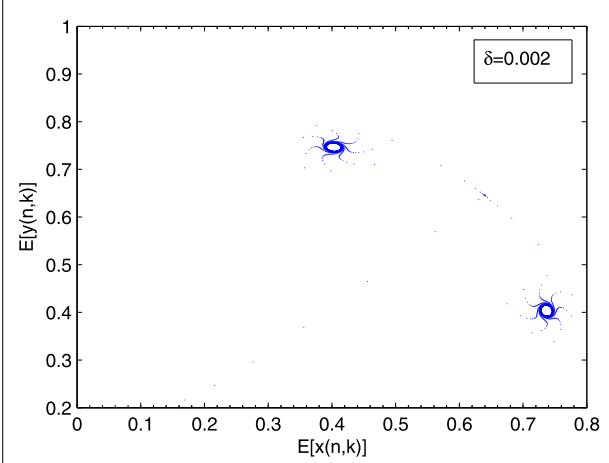

(c)

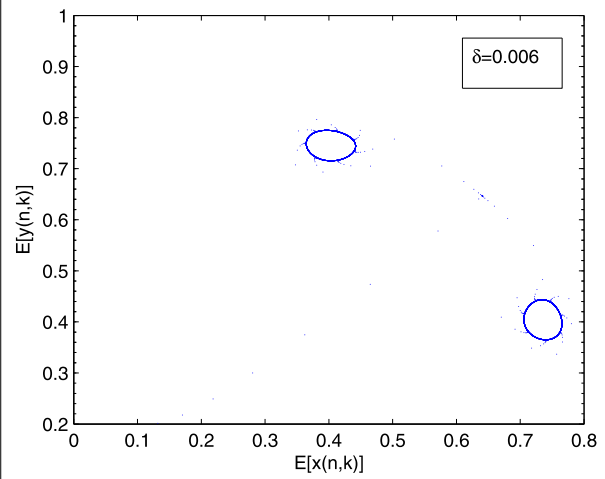

(e)

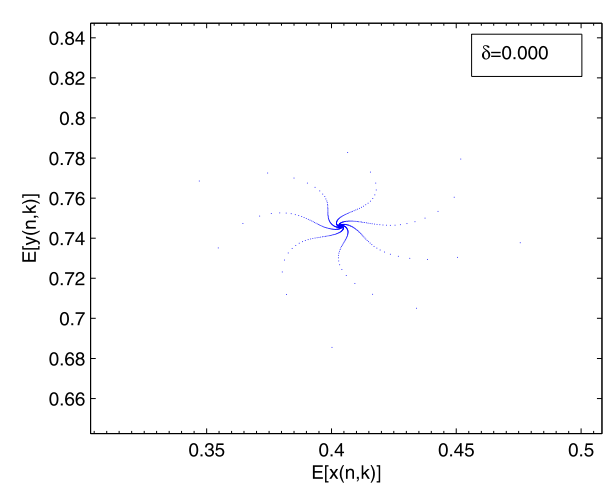

(b)

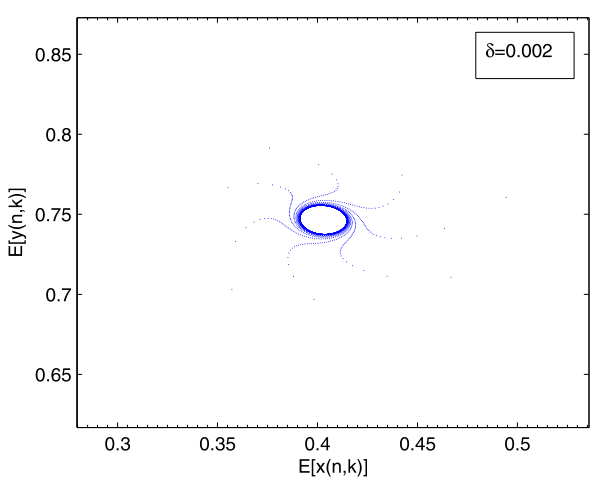

(d)

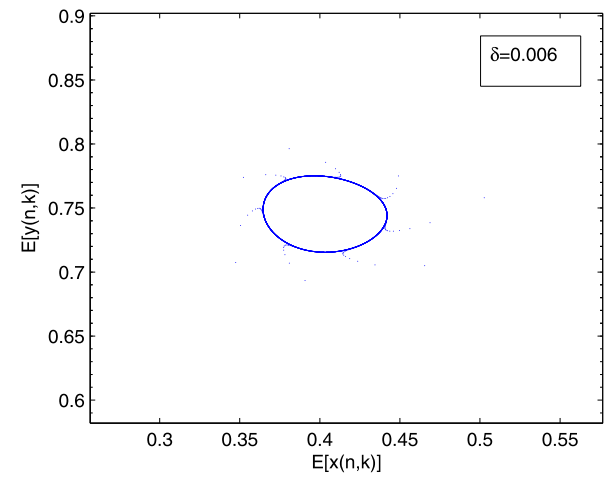

(f)

Figure 2 Phase portraits $(\mathbf{a}),(\mathbf{b}),(\mathbf{c}),(\mathbf{d}),(\mathbf{e}),(\mathbf{f}),(\mathbf{g}),(\mathbf{h}),(\mathbf{i}),(\mathbf{j}),(\mathbf{k}),(\mathbf{l}),(\mathbf{m}),(\mathbf{n}),(\mathbf{o})$ with different strength of random

appears in advance and frequency locking phenomenon occurs. In other words, within a certain range of random intensity, two-species coupled logistic system with symbiotic interaction will never die out.

\section{Conclusions}

The orthogonal polynomial approximation theory of discrete random function is applied to research the Hopf-Hopf bifurcation of the two-species discrete coupling stochastic logistic system with symbiotic interaction. Analysis shows that the orthogonal polynomial 


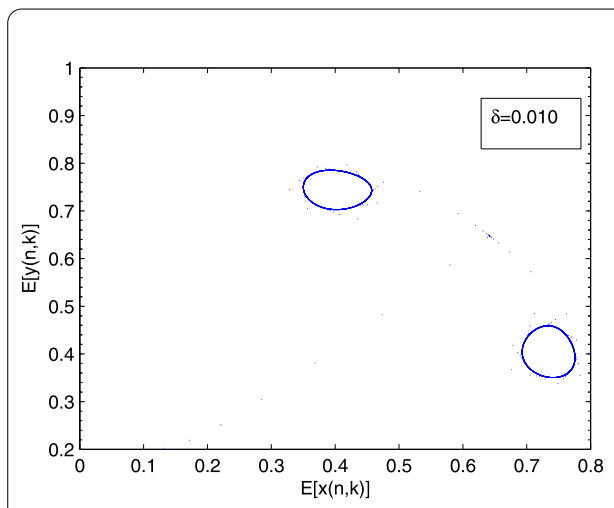

(g)

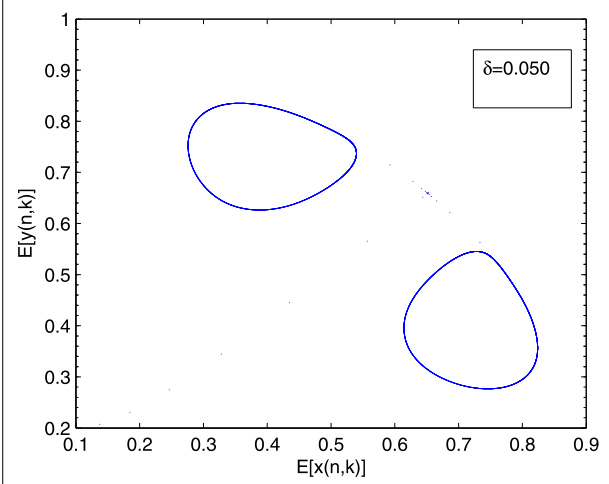

(i)

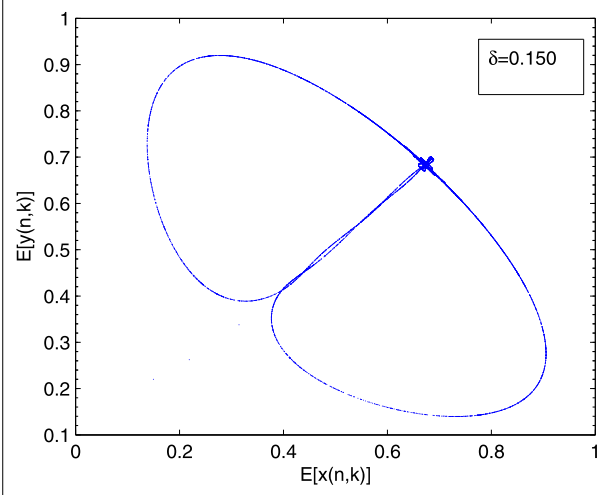

(k)

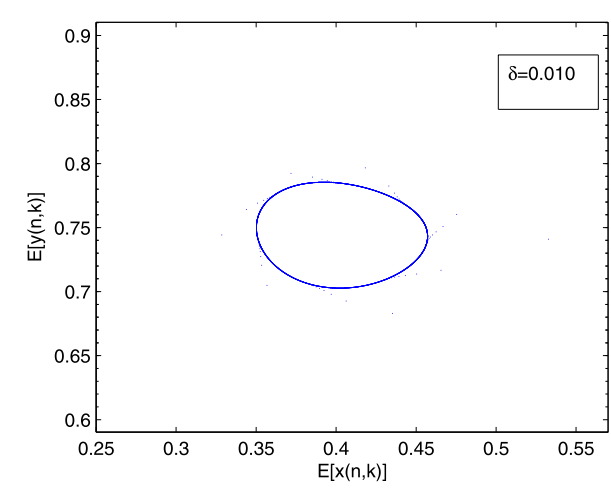

(h)

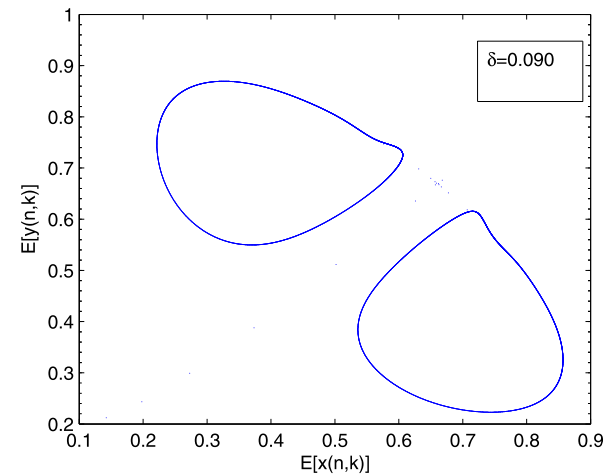

(j)

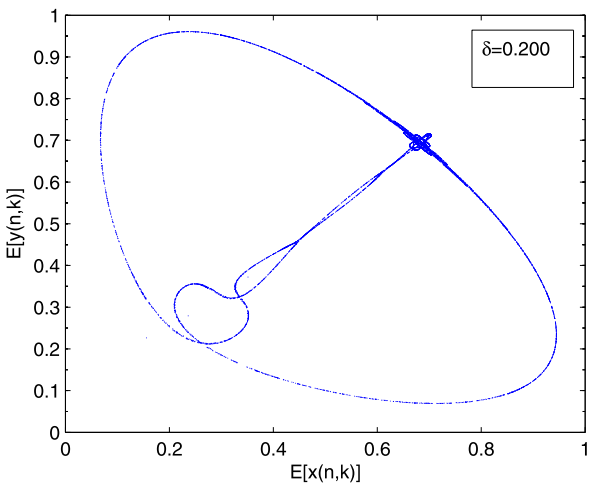

(l)

Figure 2 Continued

approximation is effective to reduce the two-species coupling logistic system with symbiotic interaction with random parameter to its equivalent deterministic system. Through the mathematical analysis and the algebraic criterion bifurcation theory, we obtain that the critical value of Hopf-Hopf bifurcation in the two-species discrete coupling stochastic logistic system with symbiotic interaction is influenced by the random intensity, and the behavior of the system varies with the random intensity. The amplitude of Hopf-Hopf bifurcation increases with the increase of random strength, and frequency locking occurs. The numerical simulation verifies the correctness of the theoretical results. Meanwhile, 


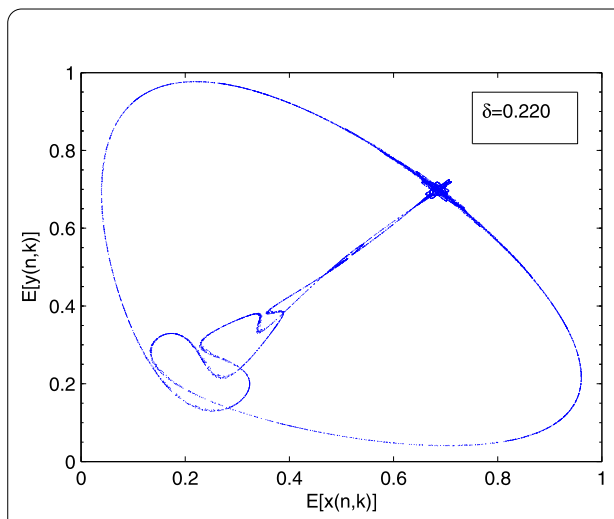

(m)

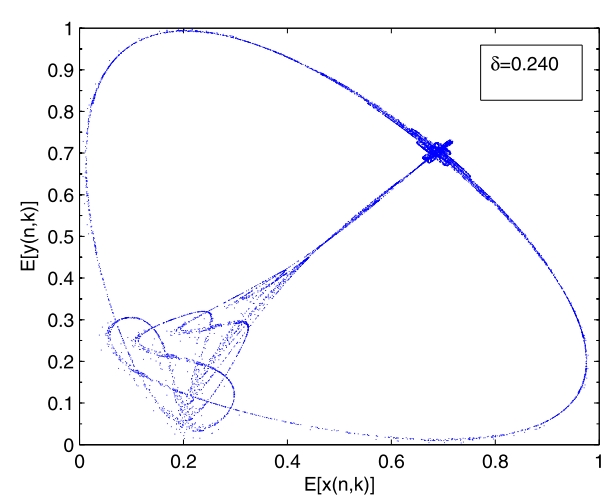

(n)

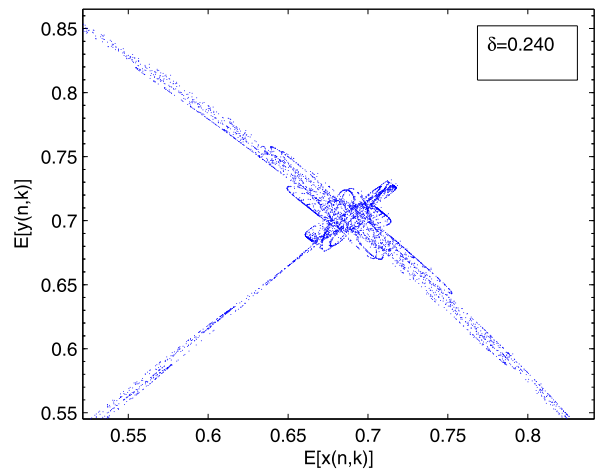

(o)

Figure 2 Continued

the discussion of numerical analysis shows that Hopf-Hopf bifurcation point appears in advance with the change of random intensity.

\section{Appendix}

$S_{i}(n), X_{i}(n), Y_{i}(n), Z_{i}(n)$, and $W_{i}(n)$ in Eq. (22) can be derived through the computer algebraic system, such as Maple, as follows:

$$
\begin{aligned}
& S_{0}(n)=x_{0}(n) y_{0}(n)+\frac{1}{2} x_{1}(n) y_{1}(n), \\
& S_{1}(n)=x_{0}(n) y_{1}(n)+x_{1}(n) y_{0}(n)+x_{1}(n) y_{1}(n), \\
& S_{2}(n)=x_{1}(n) y_{1}(n) \\
& X_{0}(n)=x_{0}^{2}(n)+\frac{1}{2} x_{1}^{2}(n), \\
& X_{1}(n)=x_{1}^{2}(n)+2 x_{0}(n) x_{1}(n), \\
& X_{2}(n)=x_{1}^{2}(n) \\
& Y_{0}(n)=y_{0}^{2}(n)+\frac{1}{2} y_{1}^{2}(n), \\
& Y_{1}(n)=y_{1}^{2}(n)+2 y_{0}(n) y_{1}(n),
\end{aligned}
$$




$$
\begin{aligned}
& Y_{2}(n)=y_{1}^{2}(n), \\
& Z_{0}(n)=x_{0}(n) y_{0}^{2}(n)+\frac{1}{2} x_{0}(n) y_{1}^{2}(n)+y_{0}(n) y_{1}(n) x_{1}(n)+\frac{1}{2} x_{1}(n) y_{1}^{2}(n), \\
& Z_{1}(n)=x_{1}(n) y_{0}^{2}(n)+2 y_{0}(n) y_{1}(n) x_{0}(n)+x_{0}(n) y_{1}^{2}(n)+2 y_{0}(n) y_{1}(n) x_{1}(n)+\frac{5}{2} x_{1}(n) y_{1}^{2}(n), \\
& Z_{2}(n)=x_{0}(n) y_{1}^{2}(n)+2 y_{0}(n) y_{1}(n) x_{1}(n)+3 x_{1}(n) y_{1}^{2}(n), \\
& Z_{3}(n)=x_{1}(n) y_{1}^{2}(n), \\
& W_{0}(n)=y_{0}(n) x_{0}^{2}(n)+\frac{1}{2} y_{0}(n) x_{1}^{2}(n)+x_{0}(n) x_{1}(n) y_{1}(n)+\frac{1}{2} y_{1}(n) x_{1}^{2}(n), \\
& W_{1}(n)=y_{1}(n) x_{0}^{2}(n)+2 x_{0}(n) x_{1}(n) y_{0}(n)+y_{0}(n) x_{1}^{2}(n)+2 x_{0}(n) x_{1}(n) y_{1}(n)+\frac{5}{2} y_{1}(n) x_{1}^{2}(n), \\
& W_{2}(n)=y_{0}(n) x_{1}^{2}(n)+2 x_{0}(n) x_{1}(n) y_{1}(n)+3 y_{1}(n) x_{1}^{2}(n), \\
& W_{3}(n)=y_{1}(n) x_{1}^{2}(n) .
\end{aligned}
$$

\section{Acknowledgements}

The authors express their sincere gratitude to the editors and referees for their helpful comments and suggestions. This work was supported by the National Natural Science Foundation (11772002), Ningxia Higher Education First-class Discipline Construction Funding Project (NXYLXK2017809), and Key Project of North Minzu University (No. 2017KJ14). The first author is partially supported by the youth project of National Open University (G16F3613Q).

\section{Funding}

This work was supported by the National Natural Science Foundation (11772002), Ningxia Higher Education First-class Discipline Construction Funding Project (NXYLXK2017809), and Key Project of North Minzu University (No. 2017KJ14). The first author is partially supported by the youth project of National Open University (G16F3613Q)

\section{Availability of data and materials}

Not applicable.

\section{Competing interests}

The authors declare that they have no competing interests.

\section{Authors' contributions}

The authors contributed equally to this paper. The authors read and approved the final manuscript.

\section{Author details}

'Department of Teaching, Guizhou Radio and TV University, Guiyang, China. ${ }^{2}$ School of Mathematics and Information Science, North Minzu University, Yinchuan, China.

\section{Publisher's Note}

Springer Nature remains neutral with regard to jurisdictional claims in published maps and institutional affiliations.

Received: 15 May 2019 Accepted: 7 June 2020 Published online: 30 June 2020

\section{References}

1. May, R.M.: Simple mathematical models with very complicated dynamics. Nature 261, 459-467 (1976)

2. Feigenbaum, M.J.: Quantitative universality for a class of nonlinear transformations. J. Stat. Phys. 19(1), 25-52 (1978)

3. Sadkowski, A.: On the application of the logistic differential equation in electrochemical dynamics. J. Electroanal. Chem. 486(1), 92-94 (2000)

4. Miskiewicz, J., Ausloos, M.: A logistic map approach to economic cycles. Phys. A, Stat. Mech. Appl. 336(1-2), 206-214 (2004)

5. Abukhamedekh, A., Besarab, D.: Development of methods of economic monitoring of the logistic system. Econ. Dev. 57(1), 20-22 (2011)

6. An, H., Searcy, S.W.: Economic and energy evaluation of a logistics system based on biomass modules. Biomass Bioenergy 46, 190-202 (2012)

7. Acevedo, M.A., Marcano, M., Fletcher, R.J.: A diffusive logistic growth model to describe forest recovery. Ecol. Model. 244, 13-19 (2012) 
8. Berezowski, M., Grabski, A.: Chaotic and non-chaotic mixed oscillations in a logistic system with delay and heat-integrated tubular chemical reactor. Chaos Solitons Fractals 14(1), 97-103 (2002)

9. Coraddu, M., Meloni, F., Mezzorani, G., Tonelli, R.: Weak insensitivity to initial conditions at the edge of chaos in the logistic map. Phys. A, Stat. Mech. Appl. 340(1-3), 234-239 (2004)

10. Dutta, D., Bhattacharjee, J.K.: Period adding bifurcation in a logistic map with memory. Phys. D: Nonlinear Phenom. 237(23), 3153-3158 (2008)

11. Masoller, C., de Souza Cavalcante, H.L.D., Leite, J.R.R.: Delayed coupling of logistic maps. Phys. Rev. E, Stat. Nonlinear Soft Matter Phys. 64, 037202 (2001)

12. He, G.-Y., He, G.-W.: Synchronous chaos in the coupled system of two logistic maps. Chaos Solitons Fractals 23(3), 909-913 (2005)

13. Jakobsen, A.: Symmetry breaking bifurcations in a circular chain of $n$ coupled logistic maps. Phys. D: Nonlinear Phenom. 237(24), 3382-3390 (2008)

14. Zhang, Y.-Q., Wang, X.-Y.: Spatiotemporal chaos in mixed linear-nonlinear coupled logistic map lattice. Phys. A, Stat. Mech. Appl. 402, 104-118 (2014)

15. Rocha, J.L., Taha, A.-K.: Allee's effect bifurcation in generalized logistic maps. Int. J. Bifurc. Chaos 29(3), 1950039 (2019)

16. Arugaslan, D., Güzel, L.: Stability of the logistic population model with generalized piecewise constant delays. Adv. Differ. Equ. 2015, 173 (2015)

17. Liu, J., Chen, L., Wei, F.: The persistence and extinction of a stochastic SIS epidemic model with logistic growth. Adv. Differ. Equ. 2018, 68 (2018)

18. Lin, Q.: Stability analysis of a single species logistic model with Allee effect and feedback control. Adv. Differ. Equ. 2018, 190 (2018)

19. Savi, M.A.: Effects of randomness on chaos and order of coupled logistic maps. Phys. Lett. A 364(5), 389-395 (2007)

20. Guo, Y.-F., Xu, W., Li, D.-X., Duan, D.-H.: Stochastic resonance in a time-delayed logistic system. Chin. J. Phys. 48(5), $683-691(2010)$

21. Xing, Z., Peng, J.: Boundedness, persistence and extinction of a stochastic non-autonomous logistic system with time delays. Appl. Math. Model. 36(8), 3379-3386 (2012)

22. Liu, Y., Liu, Q., Liu, Z.: Dynamical behaviors of a stochastic delay logistic system with impulsive toxicant input in a polluted environment. J. Theor. Biol. 329, 1-5 (2013)

23. Liu, M., Wang, K., Hong, Q.: Stability of a stochastic logistic model with distributed delay. Math. Comput. Model. 57(5-6), 1112-1121 (2013)

24. Xu, Y., Ma, S.J., Zhang, H.: Hopf bifurcation control for stochastic dynamical system with nonlinear random feedback method. Nonlinear Dyn. 65(1-2), 77-84 (2011)

25. Xu, Y., Ma, S., Zhang, H.: The stability analysis in stochastic Brusselator system with random parameter. Sci. China, Phys. Mech. Astron. 41(10), 1203-1209 (2011)

26. Liu, Q., Xu, Y., Xu, C., Kurths, J.: The sliding mode control for an airfoil system driven by harmonic and colored Gaussian noise excitations. Appl. Math. Model. 64, 249-264 (2018)

27. Ma, J., Xu, Y., Xu, W., Li, Y., Kurths, J.: Slowing down critical transitions via Gaussian white noise and periodic force. Sci. China, Technol. Sci. 62(12), 2144-2152 (2019)

28. Liu, Q., Xu, Y., Kurths, J.: Bistability and stochastic jumps in an airfoil system with viscoelastic material property and random fluctuations. Commun. Nonlinear Sci. Numer. Simul. 84, 105184 (2020)

29. Zhang, X., Xu, Y., Liu, Q., Kurths, J.: Rate-dependent tipping-delay phenomenon in a thermoacoustic system with colored noise. Sci. China, Technol. Sci. (2020). https://doi.org/10.1007/s11431-020-1589-x

30. Ma, S.J., Xu, W.: Period-doubling bifurcation in an extended Van der Pol system with bounded random parameter. Commun. Nonlinear Sci. Numer. Simul. 13, 2256-2265 (2008)

31. Xu, Y., Wang, H., Liu, D., Huang, H.: Sliding mode control of a class of fractional chaotic systems in the presence of parameter perturbations. J. Vib. Control 21, 435-448 (2015)

32. Ma, J.Z., Xu, Y., Xu, W., Li, Y., Kurths, J.: Slowing down critical transitions via Gaussian white noise and periodic force. Sci. China, Technol. Sci. 62, 2144-2152 (2019)

33. Fang, T., Leng, X.L., Song, C.Q.: Chebyshev polynomial approximation for dynamical response problem of random system. J. Sound Vib. 226, 198-206 (2003)

34. Ma, S.: The stochastic Hopf bifurcation analysis in Brusselator system with random parameter. Appl. Math. Comput. 219, 306-319 (2012)

35. Ma, S., Dong, D., Zheng, J.: Generalized synchronization of stochastic discrete chaotic system with Poisson distribution coefficient. Discrete Dyn. Nat. Soc. 2013, 981503 (2013)

36. Ma, S., Dong, D.: The asymptotic stability analysis in stochastic logistic model with Poisson growth coefficient. Theor. Appl. Mech. Lett. 4(1), 013004 (2014)

37. Fournier Prunaret, D., Lopez-Ruiz, R.: Basin bifurcations in a two-dimensional logistic map. Grazer Math. Ber. 346 123-136 (2004)

38. Boccaletti, S., Kurths, J., Osipov, G., Valladares, D.L., Zhou, C.S.: The synchronization of chaotic systems. Phys. Rep. 366 $1-101(2002)$

39. Collet, P., Eckmann, J.P.: Iterated Maps on the Interval as Dynamical Systems. Birkhäuser, Basel (1980)

40. De Sousa Vieira, M., Lichtenberg, A.J., Lieberman, M.A.: Nonlinear dynamics of self-synchronizing systems. Int. J. Bifurc. Chaos 1(3), 691-699 (1991)

41. Eckmann, J.P.: Roads to turbulence in dissipative dynamical systems. Rev. Mod. Phys. 53, 643-654 (1981)

42. Lopez-Ruiz, R., Fournier-Prunaret, D.: Complex behavior in a discrete coupled logistic model for the symbiotic interaction of two species. Math. Biosci. Eng. 1(2), 307-324 (2004)

43. Borwein, P., Erdélyi, T.: Polynomials and Polynomial Inequality. Springer, New York (1995)

44. Xu, H., Wen, G., Qin, Q., Zhou, H.: New explicit critical criterion of Hopf-Hopf bifurcation in a general discrete time system. Commun. Nonlinear Sci. Numer. Simul. 18, 2120-2128 (2013) 